A C G

publications

Rec. Nat. Prod. 15:3 (2021) 148-168

records of natural

products

\title{
Phytochemistry, Pharmacological Potency, and Potential Toxicity of Myoporum spp.
}

\author{
Arafa Musa ${ }^{1,2 *}$ \\ ${ }^{1}$ Department of Pharmacognosy, College of Pharmacy, Jouf University, 72341, Sakaka, Saudi Arabia \\ ${ }^{2}$ Department of Pharmacognosy, Faculty of Pharmacy, Al-Azhar University, Cairo, 11371, Egypt
}

(Received October 03, 2020; Revised October 23, 2020; Accepted October 24, 2020)

\begin{abstract}
Genus Myoporum family Myoporaceae, includes approximately 32 species of woody small trees or shrubs, most of them are native to Australia and surrounding territories. Only certain species have been thoroughly studied and rich in flavonoids, phenylethanoids, Phenylpropanoids, terpenoids, iridoids, essential oil, and trace alkaloids. The essential oils are characterized by sesquiterpenes type components, either in ketone or alcoholic forms usually combined to a furanoid moiety. Myoporum spp. have been utilized in folk medicine for treatment of various diseases and were used as antidermatitis, antibacterial, antipyretic, anti-pulpitis, antipsychotic, anti-inflammatory, detoxicant, and others. Despite all these benefits, Myoporum spp. must be cautiously employed due to their potential toxicities, which arise from the presence of furanosesquiterpenoid contents, particularly in their essential oil. The toxicity influences liver and can extend to kidney and lung causing injury. The present review aims to explore the phytochemistry, beneficial uses and the toxic potentials of Myoporum spp.
\end{abstract}

Keywords: Myoporum; Myoporaceae; secondary metabolites; furanosesquiterpenoids; biological activities; toxicity. (C) 2020 ACG Publications. All rights reserved.

\section{Introduction}

Family Myoporaceae is slightly a small family, consisting mainly of three genera; Bontia, Eremophila, and Myoporum. Myoporaceous plants are shrubs or small trees widely endemic to Australia. Genus Myoporum was discovered in 1786 by George Foster [1-3]. It is a widely distributed genus including approximately 30 species as reported by Chinnock and Grady [4-7], 31 species as stated by Richmond and Ghisalberti [8], or 32 species as claimed by Laurence [9-11]. It is distributed mainly in Australia, extended to Melanesia, the Pacific and Indian Ocean areas, New Guinea, Mauritius, New Zealand, and Eastern Asia. About 18 species of Myoporum are native and endemic to Australia, while the others are distributed to various regions and territories. For example, M. boninense Koidz. and M. bontioides (Sieb. et Zucc.) A. Gray is native to Japan, where they are regarded as endangered plants [12,13]. Like all Myoporaceous members, Myoporum species are rich in flavonoids; and phenylethanoids; terpenoids, iridoids and alkaloid contents [3,12-19]. Besides these secondary metabolites, most of myoporum species are characterized by secretory ducts that secrete essential oil mixture. The best known of which are the sesquiterpenes with or without furanoid moiety in their structures, which may be of ketone or alcohol. Although Myoporum spp. have been reported for their toxicity, most plant members have acquired a good reputation by aborigines [20]. The essential oil of

*E-Mail: arafa_1998@yahoo.de 
Table 1. List of Myoporum species, common names/ growing areas

\begin{tabular}{|c|c|c|c|}
\hline No. & Myoporum spp. & Common name / Area & References \\
\hline 1. & M. acuminatum R.Br. & pointed boobialla / Australia & [4] \\
\hline 2. & M. bateae F.Muell. & New South Wales & \\
\hline 3. & M. betcheanum L.S.Sm. & $\begin{array}{l}\text { mountain boobialla / New South } \\
\text { Wales, Melanesia and Queensland }\end{array}$ & \\
\hline 4. & M. boninense Koidz. & $\begin{array}{l}\text { New South Wales, Queensland, } \\
\text { Bonin to Mariana Islands }\end{array}$ & \\
\hline 5. & M. brevipes Benth. & $\begin{array}{l}\text { pale myoporum / mainly South } \\
\text { Australia }\end{array}$ & \\
\hline 6. & M. caprarioides Benth. & $\begin{array}{l}\text { slender myoporum / Western } \\
\text { Australia }\end{array}$ & \\
\hline 7. & $\begin{array}{l}\text { M. cordifolium (F.Muell.) } \\
\text { Druce }\end{array}$ & $\begin{array}{l}\text { Jerramungup myoporum / Western } \\
\text { Australia }\end{array}$ & \\
\hline 8. & M. crassifolium G.Forst. & New Caledonia, Vanuatu & \\
\hline 9. & $\begin{array}{l}\text { M. degeneri (G.L.Webster) } \\
\text { O.Deg. \& I.Deg. }\end{array}$ & Maui/Hawaii & \\
\hline 10. & $\begin{array}{l}\text { M. floribundum A.Cunn. ex } \\
\text { Benth. }\end{array}$ & $\begin{array}{l}\text { weeping boobialla / New South } \\
\text { Wales, (Victoria) }\end{array}$ & \\
\hline 11. & M. insulare R.Br. & boobialla, blueberry tree / Australia & \\
\hline 12. & M. laetum G.Forst. & Ngaio / New Zealand & \\
\hline 13. & M. mauritianum A.DC. & Mauritius and Rodrigues & \\
\hline 14. & M. montanum R.Br. & $\begin{array}{l}\text { native myrtle / Australia, New } \\
\text { Guinea and Timor }\end{array}$ & \\
\hline 15. & M. obscurum Endl. & $\begin{array}{l}\text { bastard ironwood, popwood / Norfolk } \\
\text { Island }\end{array}$ & \\
\hline 16. & M. oppositifolium R.Br. & twin-leaf myoporum / Australia & \\
\hline 17. & M. parvifolium R.Br. & creeping boobialla / Australia & \\
\hline 18. & M. petiolatum R.J.Chinnock & sticky boobialla / Australia & \\
\hline 19. & M. platycarpum R.Br. & sugar wood (Australia) & \\
\hline 20. & M. rapense $\mathrm{F} . \mathrm{Br}$. & $\begin{array}{l}\text { French Polynesia \& Kermadec island } \\
\text { in New Zealand }\end{array}$ & \\
\hline 21. & M. rimatarensis F.Br. & French Polynesia & \\
\hline 22. & M. sandwicense A.Gray & naio / Hawaii and Mangaia & \\
\hline 23. & $\begin{array}{l}\text { M. stellatum (G.L.Webster) } \\
\text { O.Deg. \& I.Deg. }\end{array}$ & O’ahu/Hawaii & \\
\hline 24. & M. stokesii F.Br. & French Polynesia & \\
\hline 25. & M. tenuifolium G.Forst. & New Caledonia and Loyalty Islands & \\
\hline 26. & M. tetrandrum (Labill.) & $\begin{array}{l}\text { Domin boobialla / coastal regions of } \\
\text { Australia }\end{array}$ & \\
\hline 27. & M. turbinatum R.J.Chinnock & salt myoporum / Western Australia & \\
\hline 28. & M. velutinum R.J.Chinnock & Western Australia & \\
\hline 29. & M. viscosum R.Br. & sticky boobialla / South Australia & \\
\hline 30. & M. wilderi Skottsb. & Cook Islands & \\
\hline 31. & $\begin{array}{l}\text { M. bontioides (Sieb. et } \\
\text { Zucc.) A. Gray A. Gray }\end{array}$ & $\begin{array}{l}\text { bitter blue plant / Northwest of } \\
\text { China, Japan }\end{array}$ & {$[12,25,28]$} \\
\hline 32. & M. deserti A. Cunn. & $\begin{array}{l}\text { Ellangowan poison bush / Japan } \\
\text { China, Australia, Hawaiian Islands, } \\
\text { New Zealand }\end{array}$ & {$[15,16,29]$} \\
\hline 33. & M. serratum $\mathrm{R}$. Br. & New Holland & {$[30,31]$} \\
\hline
\end{tabular}

M. crassifolium is incorporated in the composition of many pharmaceutical preparations employed in skin disorders associated with inflammation resulting from pruritus, sunburns, acne, eczema, redness, psoriasis, improving the signs of aging and skin scars [21,22]. Owing to the easily burning of their inner wood, some of Myoporum plants e.g. M. crassifolium, was used as a lighter and fire source on 
beaches. The small cut sticks and laths of $M$. crassifolium and M. platycarpum are also employed as candles or torches in ceremonies in the New Caledonia southern coast and Australia [13].

The Australian youths have used the resin of $M$. platycarpum to reach manhood $[4,23]$. The wood of M. laetum and M. sandwicenes are used as timber in New Zealand Hawaiian [4,10]. $M$. bontioides, has been used in folk medicine as antidermatitis, anti-bacterial, antipyretic, anti-pulpitis, antipsychotic, relieving restlessness, anti-inflammatory, treating sciatica and detoxicant $[4,17,24,25]$ in Northwest China. Additionally, Myoporum spp. can be used as a moisturizing agent in cosmetic preparations [24]. In Australia, the decoction of M. montanum leaves was used by the aboriginal people as a medicinal wash. In contrast the smoking branches for management of general ailments [4], the aqueous leaves extract has been used in respiratory and gastrointestinal disorders, laxative, analgesic in headache, an antidote for poisons and in venereal diseases [4,10,20]. Generally, Myoporum species were employed as horticultural plants, ornamental shelterbelts on roadsides to break winds [10]. In New Zealand, the juice of $M$. laetum leaves has been used as insecticide while the outer bark was applied for curing skin ulcers and eruptions. The oils of M. laetum leaves were used for septic wounds [4].

The survey stated that the nutritional value of Myoporaceous plants is limited; the fruits of $M$. montanum, and the sugary manna of $M$. platycarpum are irregularly eaten [4,8]. The aboriginal people have applied M. montanum, M. tenuifolium, M. sandwicense leaves, and the inner bark of M. laetum decoctions to relieve toothache $[4,10]$. Biological evaluation of Myoporum species revealed that they possess various biological impacts as anticancer, antibacterial, insecticidal and anti-inflammatory [24]. $M$. laetum exhibited mosquitos' repellent effect while $M$. desertii was considered insect pests deterrent with high activity against locusts [26]. M. bontioides extract displayed a strong pesticidal, insectrepellent, and antifeedant activities [26]. M. laetum possesses anti-quorum sensing (anti-QS) activity against the Chromobacterium violaceum [27].

The Myoporum essential oil particularly those with epingaione content as in M. bontioides, exhibited potent insecticidal effect against grasshoppers, Pieris rapae and leaf-cutting ants [10]. Chinnock has mentioned thirty species of Myoporum with their common names and area of distribution [4]. Additional four species have been entitled in various articles and were listed in Table 1. This review aims to explore the phytochemistry, biological activities, and potential toxicity of Myoporum spp.

\section{Biological Activities of Myoporum spp. Secondary Metabolites}

Various biological and phytochemical studies on Myoporum spp. have been performed, either to support the folk medicinal uses or to warn the aboriginals from their abuse. Generally, the Furanosesquiterpenoids of Myoporum are found to have insecticidal metabolites against the leaf-cutting ants. Unfortunately, the previous biological investigations of Myoporum spp. are scarce and have been focused mainly on certain spp. concerning $M$. bontioides, $M$. montanum, $M$. insulare, M. laetum, $M$. acuminatum and $M$. crassifolium. M. bontioides has been extensively investigated, the extract of which possessed a strong repellent activity against Plutella xylostella, and antifungal effect against Fusarium oxysporum, Sphaceloma fawcettii, Thielaviopsis paradoxa, Mycosphaerella sentina, Colletotrichum musae, Pestalotia mangiferae and Alternaria alternata [26,28,32], due to the presence of (-)-epingaione. It also exhibited anti-methicillin resistant Staphylococcus aureus (anti-MRSA) activity, the isolated sesquiterpene alkaloids (Myoporumine A and B) as well as epingaione and dehydroepingaione of $M$. bontioides also exhibited potent anti-MRSA effect with MIC values close to that of the standard vancomycin [17]. Additionally, the flavones of M. bontioides including 3,4dimethoxy-3 ,5,7-trihydroxyflavone exhibited potent antiproliferative effect against breast cancer cell line MCF-7 [24], While (2R,3R)-3,5,7-trihydroxyflavanone-3-acetate showed potent antifungal activity against Magnaporthe grisea [33]. Moreover, the essential oil and ethyl acetate fraction of which showed potent antifeedant and larvicidal activities against Spodoptera litura $[34,35]$. The plant also was incorporated in herbal mixture to treat intractable headache, sciatica and upper respiratory tract infection [36-38]. The aqueous extract M. montanum leaves exhibited significant antibacterial activity against Staphylococcus epidermidis, Enterococcus faecalis, and Moraxella catarrhalis, due to 
presence of (-)-10,11-dehydromyoporone, ( \pm -myoporone and 11-hydroxymyoporone [20]. The serrulatane diterpenes content of $M$. insulare exhibited significant anticancer activity against various types of cell lines [34,35]. The essential oil of $M$. laetum leaves showed antiviral, antimicrobial and antifungal activities $[39,40]$. The essential oil of $M$. acuminatum leaves and fruits displayed promising antimicrobial activity against G+ve bacteria: Bacillus subtilis and Streptococcus pneumonia, G-ve bacteria: E. coli and fungi: Syncephalastrum racemosum, Aspergillus fumigates and Geotricum candidum $[34,41]$. The resinous content of $M$. crassifolium was incorporated in pharmaceutical creams to treat skin disorders and inflammation [16].

\section{Secondary Metabolites Isolated from Myoporum}

Myoporum spp. are well known by various secondary metabolites contents including flavonoids, phenylethanoids, Phenylpropanoids, terpenoids, iridoids, trace alkaloids and essential oil $[12,13,15-17,42]$. the oils are characterized by sesquiterpenes type compounds, either as ketone or alcoholic forms, usually combined to a furanoid moiety, to which the toxicity of Myoporum was attributed [20]. The essential oil is nearly distributed in all Myoporaceous plants. Flavonoids have been isolated from $M$. tenuifolium, M. deserti, M. acuminatum, M. bontioides and M. serratum. Organic acids and phenylethanoids have been obtained from $M$. bontioides. Serrulatane type diterpene were isolated from $M$. insulare. Iridoid glycosides have been isolated from $M$. bontioides, $M$. deserti and $M$. insulare. $\beta$-sitosterol and the sesquiterpene alkaloids (myoporumine $\mathrm{A}$ and $\mathrm{B}$ ) were detected and isolated from $M$. bontioides, while the furanosesquiterpenoids have been isolated from most of myoporum spp. as mentioned in Table 2 and chemical structures of the compounds are given Figure 1. [12,28,43-45].

Table 2. Secondary metabolites isolated from Myoporum spp.

\begin{tabular}{|c|c|c|c|}
\hline No & Compound name & Distribution / Plant name & References \\
\hline \multicolumn{4}{|c|}{ Flavones } \\
\hline 1. & Apigenin & $\begin{array}{l}\text { M. bontioides \& M. tenuifolium } \\
\text { leaves }\end{array}$ & {$[12,28,45]$} \\
\hline 2. & Apigenin-7-O-rutinoside & M. tenuifolium leaves & {$[45]$} \\
\hline 3. & Apigenin-7-O- glucoside & M. bontioides leaves & {$[12,44]$} \\
\hline 4. & Apigenin-7-O-glucuronide & M. bontioides leaves and flowers & {$[12]$} \\
\hline 5. & $\begin{array}{l}(1 \rightarrow 6) \text {-rhamnosyl-6-amino-6- } \\
\text { deoxyglucosyl-7-O-apigenin. }\end{array}$ & M. tenuifolium leaves & {$[8,50]$} \\
\hline 6. & chrysoeriol & $\begin{array}{l}\text { M. bontioides Gray leaves } \\
\text { M. tenuifolium leaves }\end{array}$ & {$[12,45]$} \\
\hline 7. & chrysoeriol-7-O-gentiobioside & M. tenuifolium leaves & {$[45,51]$} \\
\hline 8. & chrysoeriol-7-O-rutinoside & M. tenuifolium leaves & {$[45,51]$} \\
\hline 9. & chrysoeriol-7-O-glucoside & M. bontioides leaves & {$[12]$} \\
\hline 10. & chrysoeriol-7-O-glucuronide & M. bontioides leaves & [12] \\
\hline 11. & $\begin{array}{l}(1 \rightarrow 6) \text {-rhamnosyl-6-amino-6- } \\
\text { deoxyglucosyl-7-O-chrysoeriol }\end{array}$ & M. tenuifolium leaves & {$[50]$} \\
\hline 12. & norartocarpetin & M. bontioides leaves acetone extract & {$[24]$} \\
\hline 13. & luteolin & $\begin{array}{l}\text { M. tenuifolium leaves } \\
\text { M. deserti } \\
\text { M. acuminatum } \\
\text { M. bontioides leaves }\end{array}$ & $\begin{array}{c}{[12,24,28,43,} \\
45,51]\end{array}$ \\
\hline 14. & luteolin-7-O-glucoside & $\begin{array}{l}\text { M. tenuifolium leaves } \\
\text { M. bontioides leaves }\end{array}$ & {$[12,45,51]$} \\
\hline 15. & luteolin-7-O-rutinoside & M. tenuifolium leaves & [45] \\
\hline 16. & luteolin-7-O-glucuronide & $\begin{array}{l}\text { M. bontioides A. Gray leaves and } \\
\text { flowers }\end{array}$ & {$[12], 107$} \\
\hline
\end{tabular}




\begin{tabular}{|c|c|c|c|}
\hline 17. & $\begin{array}{l}\text { (1-6)-glucosyl-6-amino-6- } \\
\text { deoxyglucosyl-7-O-chrysoeriol }\end{array}$ & M. bontioides leaves & {$[52]$} \\
\hline 18. & $\begin{array}{l}\text { (1-6)-glucosyl-6-amino-6- } \\
\text { deoxyglucosyl-7-O-luteolin }\end{array}$ & M. bontioides leaves & [52] \\
\hline 19. & $\begin{array}{l}\text { 5-hydroxy- } 6,7,3^{\prime}, 4^{\prime}- \\
\text { tetramethoxyflavone }\end{array}$ & M. bontioides leaves and flowers & [28] \\
\hline 20. & Tricin & M. tenuifolium leaves & {$[24,45]$} \\
\hline 21. & tricin-7-O-glucuronide & $\begin{array}{l}\text { M. tenuifolium leaves } \\
\text { M. bontioides }\end{array}$ & {$[12,45]$} \\
\hline 22. & diosmetin & M. bontioides leaves acetone extract & {$[24]$} \\
\hline 23. & tangeretin & M. bontioides leaves & [28] \\
\hline 24. & sinensetin & M. bontioides leaves & [28] \\
\hline 25. & Nobiletin & M. bontioides leaves & [28] \\
\hline 26. & 5-demethylnobiletin & M. bontioides leaves & [28] \\
\hline 27. & $\begin{array}{l}5,4^{\prime} \text {-dihydroxy- } 6,7,8,3^{\prime}- \\
\text { tetramethoxy flavone }\end{array}$ & M. bontioides leaves & [28] \\
\hline 28. & 4',5,7,8-pentamethoxyflavone & M. bontioides leaves & [28] \\
\hline 29. & selagin-7- $O$-glucoside & M. bontioides leaves & [12] \\
\hline 30. & selagin-7-O-glucuronide & M. bontioides leaves & [12] \\
\hline 31. & $\begin{array}{l}\text { 3,4-dimethoxy-3`,5,7- } \\
\text { trihydroxyflavone }\end{array}$ & M. bontioides leaves & {$[24,43]$} \\
\hline \multicolumn{4}{|c|}{ Flavonols } \\
\hline 32. & galangin & M. bontioides & {$[12,53]$} \\
\hline 33. & galangin-3-methylether & M. bontioides & [53] \\
\hline 34. & ermanin & M. bontioides & [12] \\
\hline 35. & quercetin-3-methyl ether & M. bontioides leaves & [12] \\
\hline 36. & quercetin-3,4-dimethyl ether & M. bontioides leaves & [12] \\
\hline 37. & 3,3'-dimethoxyquercetin & M. bontioides leaves acetone extract & [24] \\
\hline 38. & Isorhamnetin & M. bontioides leaves & [43] \\
\hline 39. & Kaempferol & M. serratum & [12] \\
\hline 40. & 3,4-dimethoxy kaempferol & M. bontioides leaves & 106 \\
\hline 41. & Dihydrokaempferol & M. bontioides leaves & [28] \\
\hline 42. & Isokaempferide & M. bontioides leaves & [12] \\
\hline 43. & rhamnocitrin & $\begin{array}{l}\text { M. bontioides leaves acetone extract } \\
\text { Flavanones }\end{array}$ & [24] \\
\hline 44. & eriodictyol-7-O-rutinoside & Myoporum tenuifolium & [45] \\
\hline 45. & $\begin{array}{l}(2 \mathrm{R}, 3 \mathrm{R})-3,5,7- \\
\text { trihydroxyflavanone } 3 \text {-acetate }\end{array}$ & M. bontioides & {$[53,54]$} \\
\hline 46. & pinocembrin & M. bontioides leaves & {$[12,53]$} \\
\hline 47. & Pinobanksin & M. bontioides leaves & [53] \\
\hline \multicolumn{4}{|c|}{ Anthocyanin } \\
\hline 49. & cyanidin-3,5-di- $O$-glucoside & M. bontioides flowers & {$[12]$} \\
\hline \multicolumn{4}{|c|}{ Organic acids, phenylpropanoids and phenylethanoids } \\
\hline 50. & $\begin{array}{l}\text { verbascoside, isoverbascoside and } \\
\text { oxoverbascoside }\end{array}$ & M. bontioides leaves and flowers & {$[12,44,55]$} \\
\hline 51. & Sesamin & M. bontioides leaves & {$[53,55]$} \\
\hline 52. & chlorogenic acid & M. bontioides leaves & {$[12]$} \\
\hline 53. & Cimidahurine & M. bontioides leaves & [44] \\
\hline 54. & Meliotoside & M. bontioides leaves & [44] \\
\hline
\end{tabular}


55. ferulic acid $\beta$-D-glucopyranosyl $\quad$ M. bontioides leaves

ester (phenylpropanoids)

\section{Serrulatane diterpenes}

56. 7,8,18-trihydroxyserrulat-14-ene Myoporum insulare resins/exudates

$[19,31,35,56]$

57. 5,18-epoxyserrulat-14-en-8,18- Myoporum insulare resins/exudates

$[19,35,57]$ diol

58. 5,18-epoxy-8-hydroxyserrulat-14- Myoporum insulare resins/exudates ene

59. 7,8-dihydroxyserrulat-14-ene

Myoporum insulare resins/exudates Myoporum insulare resins/exudates

61. 5,18-epoxyserrulat-14-en-7,8Myoporum insulare resins/exudates dione

62. $\beta$-sitosterol

Steroids

63. Stigmasterol

$M$. bontioides leaves acetone extract

M. bontioides leaves

\section{Sesquiterpene alkaloids}

64. Myoporumine A acetone fraction of $M$. bontioides

65. Myoporumine B acetone fraction of $M$. bontioides

\section{Iridoids}

66. Myopochlorin

M. bontioides

67. Myobontioside A \& B

M. bontioides

68. (1R)-1-methoxymyodesert-3-ene

M. deserti essential oil

69. (+)-(1R)-1-acetoxymyodesert-3-

M. deserti essential oil ene

70. (-)-(1S)-1-acetoxymyodesert-3ene

M. deserti essential oil

71. Harpagide

M. insulare

72. 8-acetylharpagide

M. bontioides

73. macfadyenoside (5-

$M$. insulare

$M$. bontioides hydroxycatalpol)

M. insulare

\section{Furanoid sesquiterpene ketones}

75. Epingaione

76. Dehydroepingaione

77. Myoporone

78. Dehydromyoporone

79. 9-(3-furanyl)-2,6-dimethyl-4nonanone

80. Dihydrocrassifolone

81. 10,11-dehydroisomyodesmone

82. 10,11-dehydromyodesmone
M. bontioides leaves acetone fraction acetone fraction of $M$. bontioides \& $M$. montanum leaves acetone fraction of $M$. bontioides \& $M$. montanum leaves

M. bontioides leaves acetone fraction

M. bontioides leaves acetone fraction acetone extract \& essential oil of $M$. montanum leaves acetone extract \& essential oil of $M$. montanum leaves 


\section{Furanoid sesquiterpene ketols isolated from Myoporum}

83. 10,11-dehydromyoporum ketol acetone extract \& essential oil of $M$.

84. myoporum ketol montanum leaves

acetone extract \& essential oil of $M$. montanum leaves

85. Crassifolone M. crassifolium essential oil

86. dihydrocrassifolone

M. crassifolium essential oil

87. (-)- epi- $\alpha$-bisabolol (-)-anymol

M. crassifolium essential oil (65.1\%)

88. Dendrolasin

M. crassifolium essential oil

89. $\alpha$-bisabolol oxide- $B$

M. crassifolium essential oil (7.3\%)

\section{Major essential oil contents}

90. Ngaione

91. (+)-myomontanone

92. (+)-10,11didehydromyomontanone

93. isomyomontanone

94. dehydrongaione

95. epingaione

96. dehydroepingaione

97. myodesmone

98. dehydromyodesmone

99. isomyodesmone

100. dehydroisomyodesmone

101. myoporone

\section{Miscellaneous}

102. myobontioside $\mathrm{C}$ (acetogenin glucoside)

103. myobontioside $\mathrm{D}$ (monoterpene glucoside)

104. Prunasin
M. deserti

M. acuminatum (leaves \& fruits)

M. laetum

M. acuminatum fruits essential oil

M. montanum ( $70 \%$ of the oil)

M. betcheanum

M. montanum

M. montanum (3\% of the oil)

M. deserti

M. deserti and M. bontioides (81.52

$\%)$

M. deserti

M. deserti

M. acuminatum

M. deserti

M. deserti

M. acuminatum

M. deserti

M. deserti

M. montanum (22\% of the oil)

M. betcheanum
$[13,29,34,40$, $58]$

$[34,46]$

$[46,59]$

$[26,29,43,60$,

$[29,46,63,64]$

M. bontioides

$M$. bontioides

M. bontioides

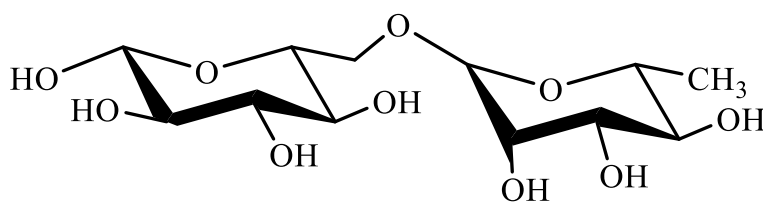

rutinoside moiety

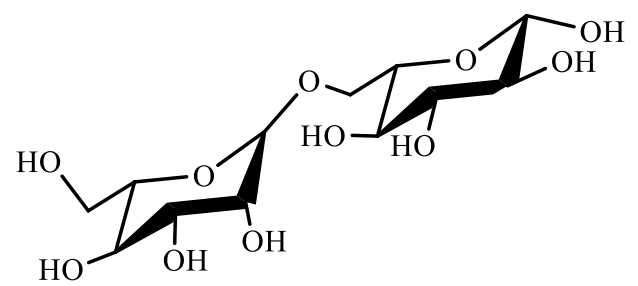

gentiobioside moiety 
<smiles>OC1C[C@H](O)[C@H](O)[C@H](O)[C@H](O)O1</smiles>

$\beta$-D-glucose moiety

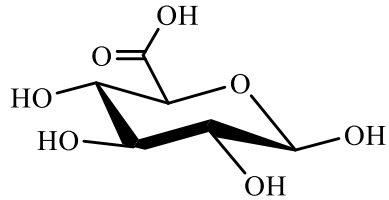

glucuronide moiety<smiles>[R20]c1cc(O)c2c(=O)cc(-c3cc([R])c(O)c([R])c3)oc2c1</smiles>

\begin{tabular}{cccc}
\hline Compound No & $\mathbf{R}$ & $\mathbf{R}_{\mathbf{1}}$ & $\mathbf{R}_{\mathbf{2}}$ \\
\hline $\mathbf{1}$ & $\mathrm{H}$ & $\mathrm{H}$ & $\mathrm{H}$ \\
$\mathbf{2}$ & $\mathrm{H}$ & $\mathrm{H}$ & rutinoside \\
$\mathbf{3}$ & $\mathrm{H}$ & $\mathrm{H}$ & $\beta$-D-glucoside \\
$\mathbf{4}$ & $\mathrm{H}$ & $\mathrm{H}$ & glucuronide \\
$\mathbf{6}$ & $\mathrm{OCH}_{3}$ & $\mathrm{H}$ & $\mathrm{H}$ \\
$\mathbf{7}$ & $\mathrm{OCH}_{3}$ & $\mathrm{H}$ & gentiobioside \\
$\mathbf{8}$ & $\mathrm{OCH}_{3}$ & $\mathrm{H}$ & rutinoside \\
$\mathbf{9}$ & $\mathrm{OCH}_{3}$ & $\mathrm{H}$ & glucoside \\
$\mathbf{1 0}$ & $\mathrm{OCH}_{3}$ & $\mathrm{H}$ & glucuronide \\
$\mathbf{1 3}$ & $\mathrm{OH}$ & $\mathrm{H}$ & $\mathrm{H}$ \\
$\mathbf{1 4}$ & $\mathrm{OH}$ & $\mathrm{H}$ & $\beta$-D-glucoside \\
$\mathbf{1 5}$ & $\mathrm{OH}$ & $\mathrm{H}$ & rutinoside \\
$\mathbf{1 6}$ & $\mathrm{OH}_{2}$ & $\mathrm{H}$ & glucuronide \\
$\mathbf{2 0}$ & $\mathrm{OCH}_{3}$ & $\mathrm{OCH}$ & $\mathrm{H}$ \\
$\mathbf{2 1}$ & $\mathrm{OCH}_{3}$ & $\mathrm{OCH}$ & glucuronide \\
$\mathbf{2 9}$ & $\mathrm{OCH}_{3}$ & $\mathrm{OH}$ & glucoside \\
$\mathbf{3 0}$ & $\mathrm{OCH}_{3}$ & $\mathrm{OH}$ & glucuronide \\
\hline
\end{tabular}

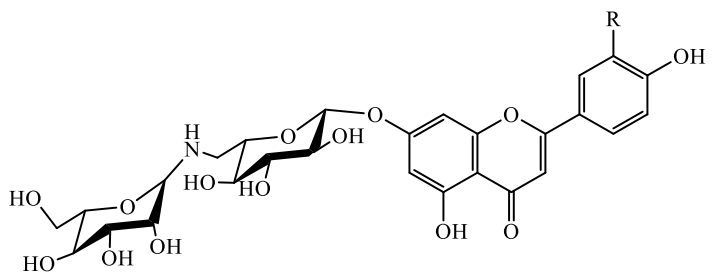

17: $\mathrm{R}=\mathrm{OCH}_{3} \quad$ 18: $\mathrm{R}=\mathrm{OH}$

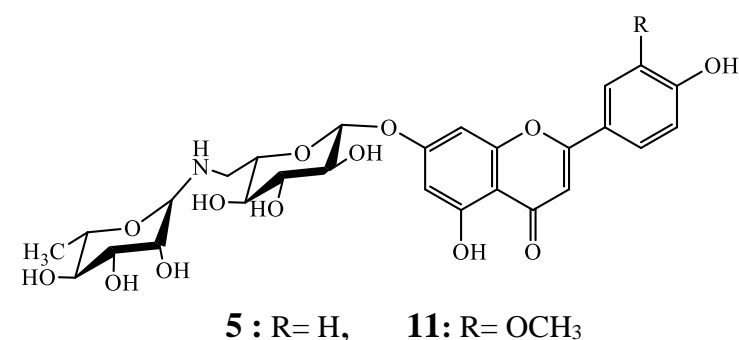




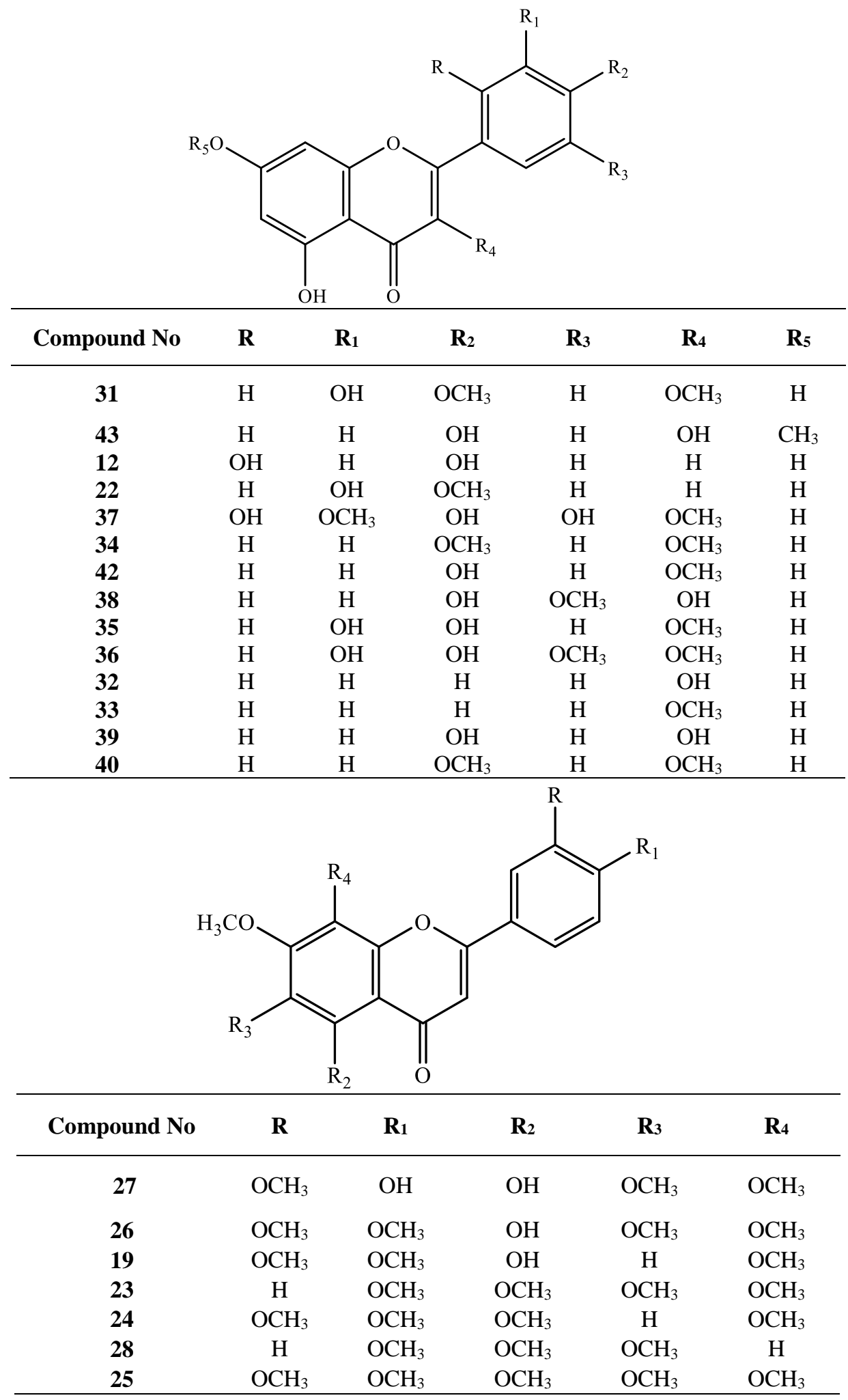




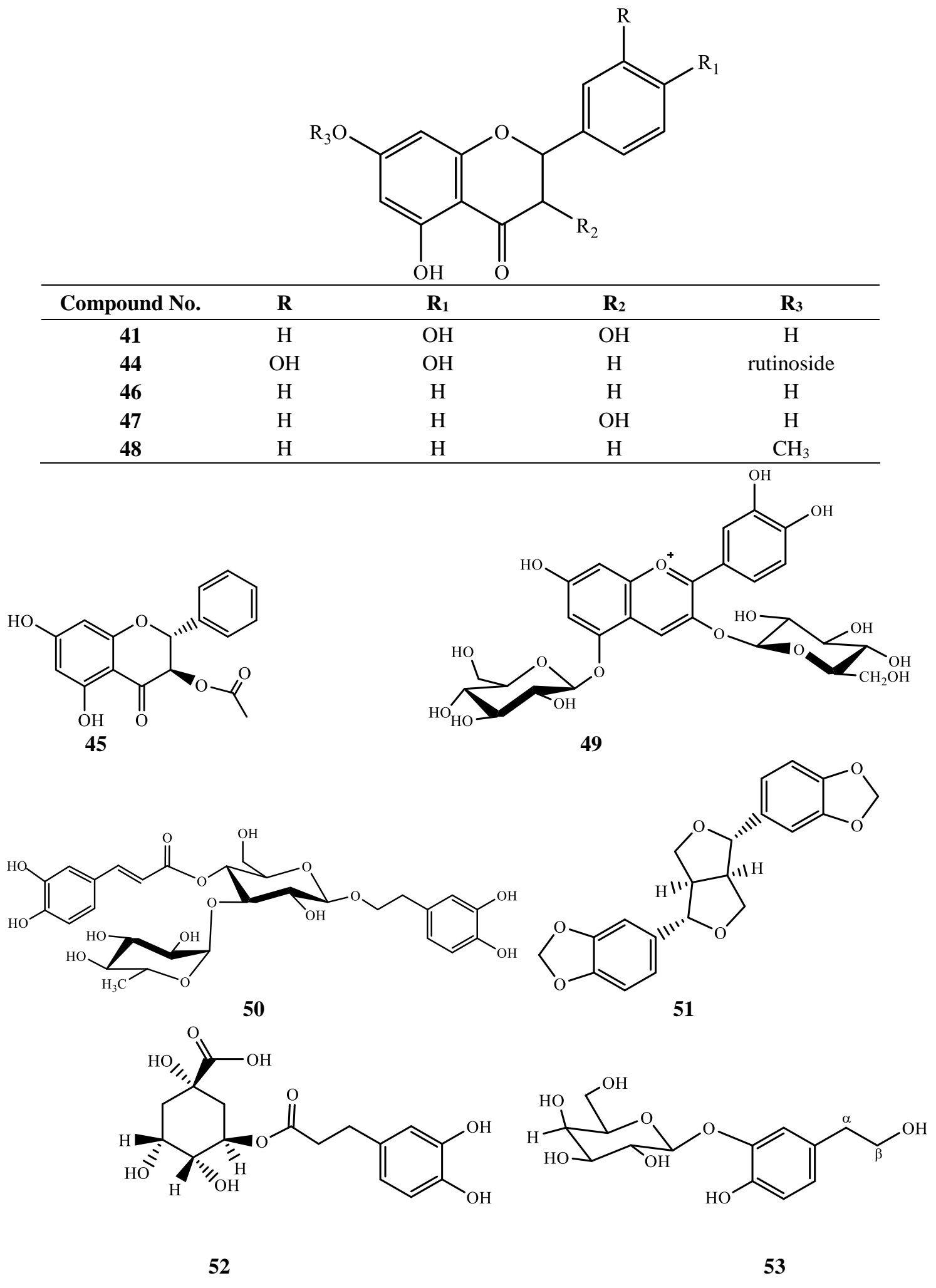


<smiles>O=C(O)/C=C/c1ccccc1O[C@@H]1O[C@H](O)[C@H](O)[C@H](O)[C@H](O)[C@H]1O</smiles><smiles>[R]C1Oc2c(C)cc(O)c3c2[C@H](CC[C@H]3C)[C@H]1CCC=C(C)C</smiles><smiles>[R]O[Na]</smiles>

61<smiles>COc1cc(/C=C/C(=O)O[C@H]2O[C@H](O)[C@@H](O)[C@H](O)[C@H](O)[C@H]2O)ccc1O</smiles><smiles>CC(C)=CCC[C@H](C)[C@H]1CC[C@@H](C)c2c1cc(C)c(O)c2O</smiles>

59<smiles>CC(C)=CCC[C@H](CO)[C@H]1CC[C@@H](C)c2c1cc(C)c(O)c2O</smiles>

56

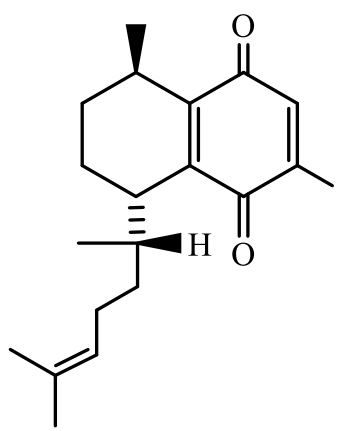

60<smiles>CCC(CC[C@H](C)C1CCC2C3CC=C4C[C@@H](O)CC[C@]4(C)C3CC[C@@]21C)C(C)C</smiles>

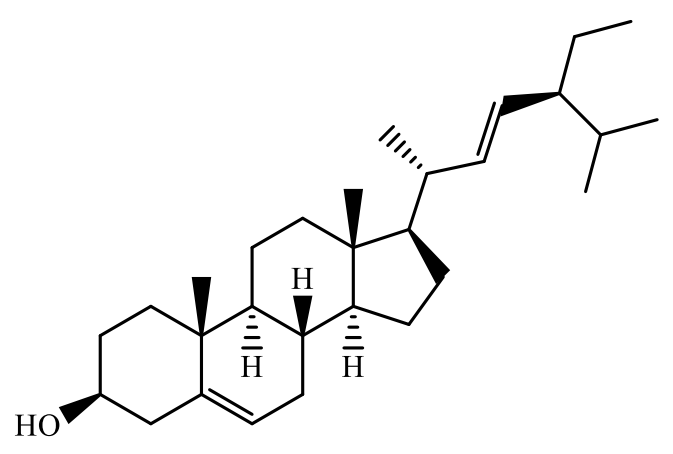

63<smiles>CC(C)CC(=O)C[C@]1(C)CC[C@H](C2=CCNC2=O)O1</smiles>

64 


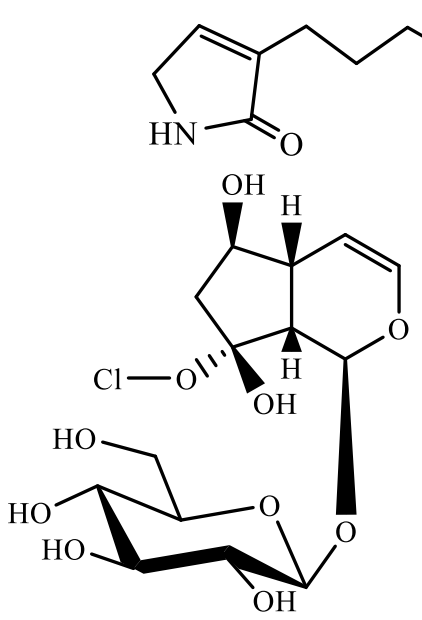

65

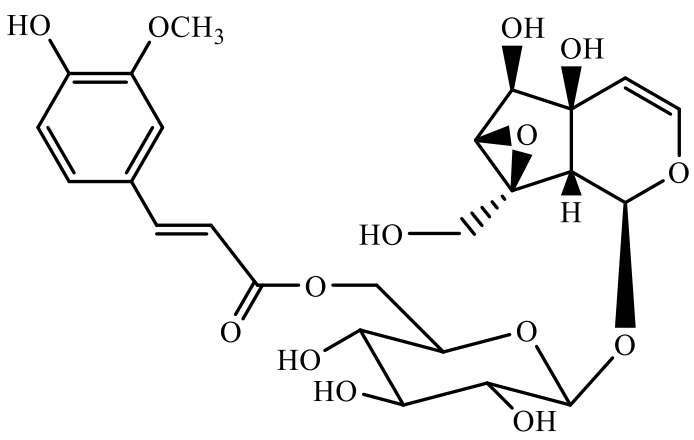

67b

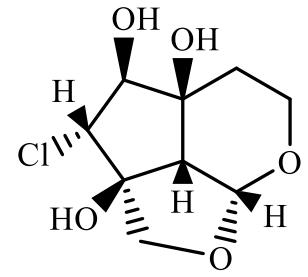

66

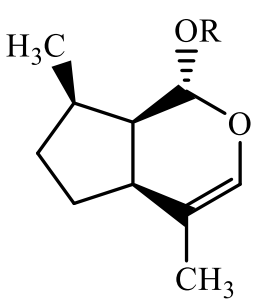

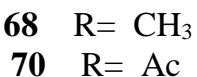

$67 a$

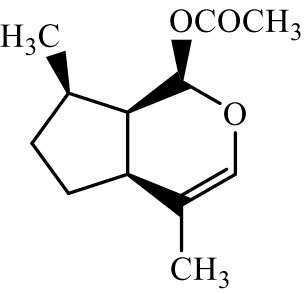

69

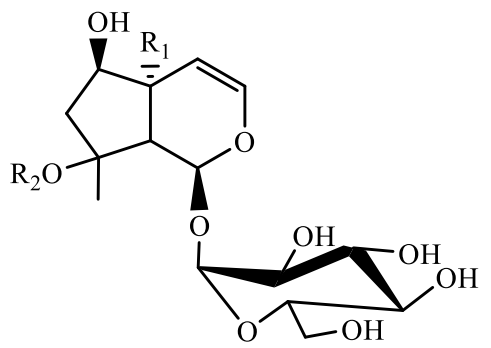

$\begin{array}{ccc} & \mathrm{R}_{1} & \mathrm{R}_{2} \\ \mathbf{7 1} & \mathrm{OH} & \mathrm{H} \\ \mathbf{7 2} & \mathrm{OH} & \mathrm{Ac} \\ \mathbf{7 4} & \mathrm{H} & \mathrm{H}\end{array}$<smiles>CC(C)=CC(=O)C[C@]1(C)CC[C@@H](c2ccoc2)O1</smiles>

96,76

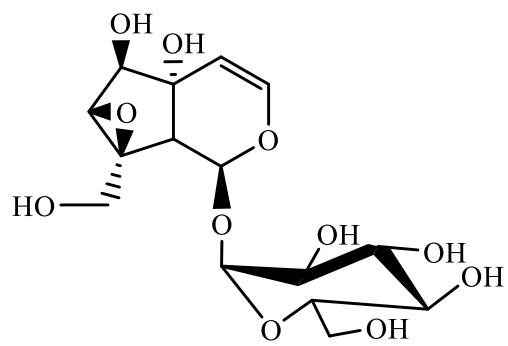

macfadyenoside $\mathbf{7 3}$<smiles>CC(C)CC(=O)CC(C)CCC(=O)c1ccoc1</smiles>

101,77

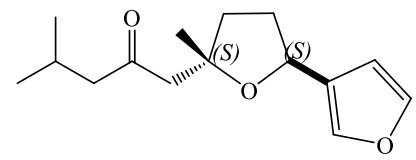

(+)-epingaione $\mathbf{9 5 , 7 5}$<smiles>CC(C)=CC(=O)C[C@@H](C)CCC(=O)c1ccoc1</smiles>

78 


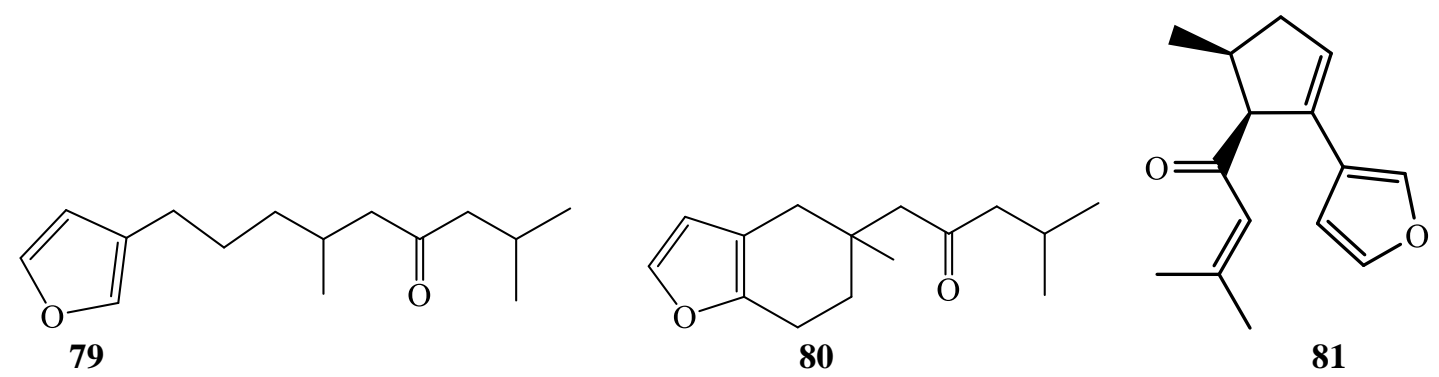

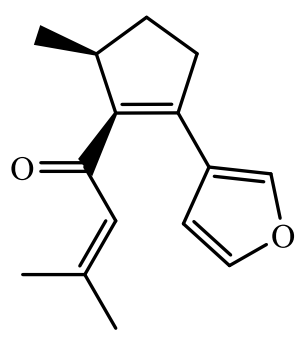

82

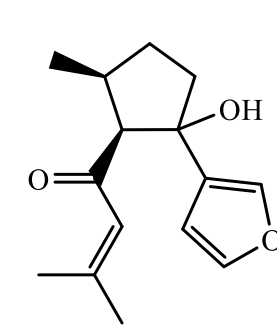

83

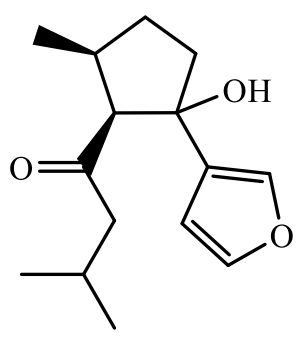

84<smiles>CC(=O)CC1(C)CCCc2ccoc21</smiles>

85<smiles>CC(=O)CC1(C)CCCc2ccoc21</smiles>

86<smiles>CC(C)=CCC[C@@](C)(O)[C@H]1CC=C(C)CC1</smiles>

87<smiles>CC(C)=CCC/C(C)=C/CCc1ccoc1</smiles>

88

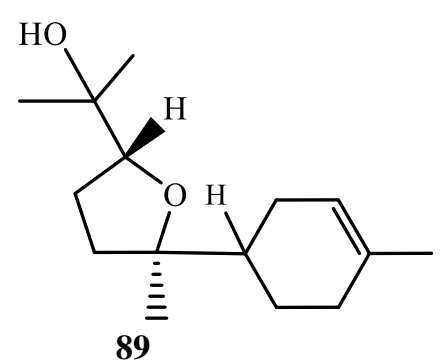<smiles>CC(C)CC(=O)C[C@@]1(C)CC[C@@H](c2ccoc2)O1</smiles>

90

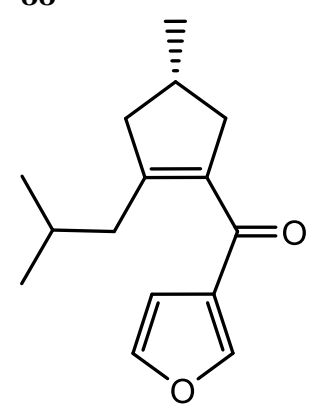

91<smiles>CC(C)=CC1=C(C(=O)c2ccoc2)C[C@H](C)C1</smiles>

92<smiles>CC(C)CC1=C[C@H](C)C[Z]1C(=O)c1ccoc1</smiles>

93<smiles>CC(C)=CC(=O)C[C@]1(C)CC[C@@H](c2ccoc2)O1</smiles>

94

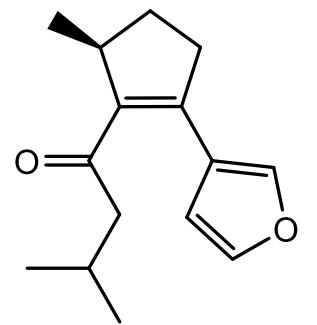

97 


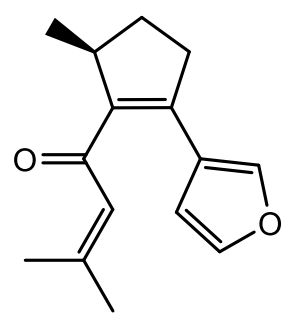

98

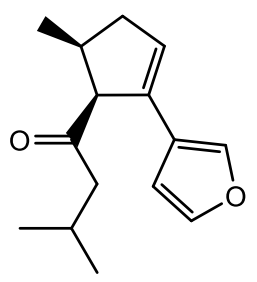

99

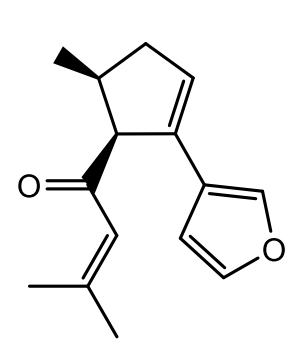

100

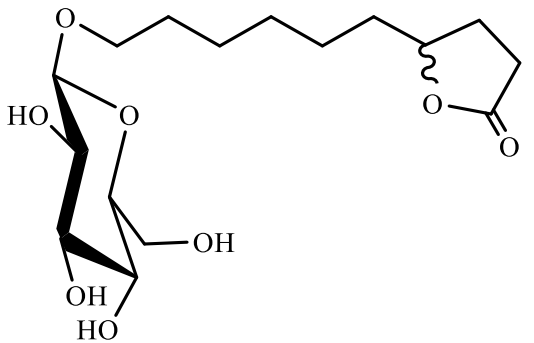

102

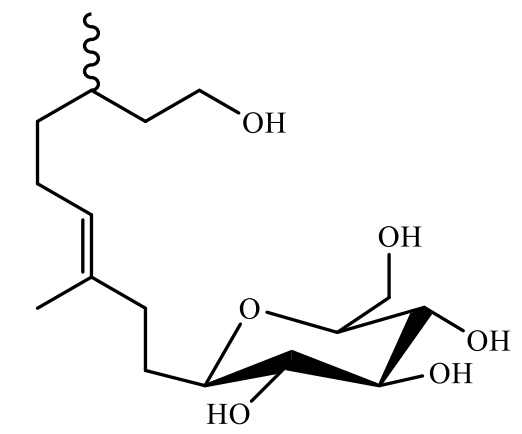

103

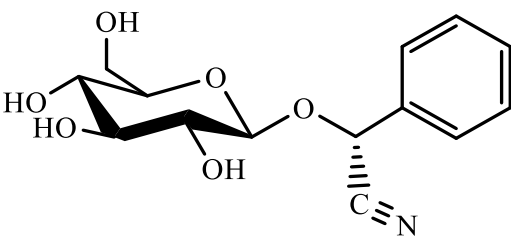

104

Figure 1. Chemical structures diversity present in Myoporum spp.

\subsection{Essential oils of Myoporum spp.}

Essential oils are nearly distributed in all Myoporaceous plants, the previous studies have focused mainly on the composition of essential oil of Myoporum leaves [4], while researches on fruits oil are scarce $[18,34]$. The oils are famous for their contents of toxic furanoid sesquiterpenes which may be of ketone (oxygenated farnesols) or alcohol types [13,40]. Furanosesquiterpenoid ketones such as $( \pm)$-myoporone, dehydromyoporone, myodesmone, dehydromyodesmone, ngaione, epingaione, dehydrongaione, dehydroepingaione were detected and isolated from M. acuminatum, M. laetum, M. tenuifolium, $M$. betcheanum, $M$. deserti, $M$. tetrandrum, $M$. crassifolium, $M$. bontioides [46-48]. The oils of $M$. acuminatum were characterized by high percentage of myoporone and myodesmone. $M$. betcheanum and $M$. maculatum were rich in myoporone as the major essential oil content, the high percentage of dehydrongaione and dehydromyodesmone were detected in $M$. deserti and $M$. maculatum. Myomontanone, isomyodesmone, dehydroisomyodesmone, dehydromyodesmone, myoporone and dehydromyoporone were detected in high percentage in $M$. montanum and $M$. acuminatum [10,21,47,49]. The other sesquiterpenoid members such as crassifolone, dihydrocrassifolone and dendrolasin were also found in M. crassifolium [13]. The essential oil of Egyptian M. laetum leaves produced the highest percentage (0.23\%) when collected in August [40]. it was rich in oxygenated sesquiterpene compounds including ngaione, elemicin, dehydromyoporone, myoporone and myomontanone in ratios of $79.63 \%, 10.74 \%, 1.92 \% 0.80 \%$ and $0.02 \%$, respectively [39]. The alcohol sesquiterpenoids were detected in the essential oil of $M$. crassifolium inner wood and M. montanum leaves [40]. M. crassifolium yielded about $1.54 \%$ essential oil, with the major component of (-)- epi- $\alpha$-bisabolol or its epimer (-)-anymol in concentration of $(65.1 \%)$, bisabolol oxide isomers B1 and B2 in concentrations of $7.3 \%$ and $9.1 \%$, respectively $[10,13,21,49]$. While $M$. montanum was rich in 10,11-dehydromyoporum ketol (2.5\%) and Myoporum ketol (3.6\%). The essential oil of $M$. deserti, consisting mainly of furanosesquiterpene ketones (-)-ngaione, (-)dehydrongaione, myodesmone, myoporone, (-)-epingaione, and (-)-dehydroepingaione [13, 29]. It also contains monoterpene iridoid, $(1 R)$-1-methoxymyodesert-3-ene which is nontoxic to the sheep and hemiacetal esters iridoid, $(+)-(1 R)$ - and (-)-(1S)-1-acetoxymyodesert-3-ene [15, 16]. It has been stated that $M$. deserti was responsible for the severe stock poisoning and the loss of numerous sheep and cattle, despite its high nutritional value as fodder $[15,16]$. 


\section{Potential Toxicity of Myoporum spp.}

Liver is the largest organ in the human belly consisting of two lobes and located at the right abdominal side. It is reddish-brown with rubbery touch and being enclosed by the rib cage $[65,66]$. The main function of the liver is associated with digestion, blood filtration, metabolism, detoxification of the body from the harmful chemicals and production of vital proteins such as albumin and others involved in blood clotting [67,68]. Hepatic disorders may arise from injury by drugs, chemicals, phytotoxins, pesticides contaminated foods and environmental pollution. The injured liver may be manifested by abdominal pain, hepatitis which is characterized by an elevation in alanine aminotransferase (ALT), alkaline phosphatase (ALP) and aspartate amino transferase (AST) levels in the blood, anorexia, nausea, fatigue, weakness, cholestasis, high bilirubin level, jaundice, icterus and itching [57,65,69-72]. So, exploring the plants risks is of great importance and is considered as significant as exploring their beneficial effects. Either to warn the populations from the hazards of their abuse, or to regulate the prescribed therapeutic doses. Additionally, the literature survey found no distinct cuts between the medicinally used plants and the toxic ones, as most of them were considered toxic with low safety index, and the toxicity may arise from the accumulation of certain metabolites by the time in the living body $[73,74]$. Most of the Myoporum essential oils are characterized by their sesquiterpenoid contents, which may be hydrocarbons or furanosesquiterpenoid ketones or alcohols. The furan-containing sesquiterpenoids are regarded as toxic phytochemicals [75-77]. The most commonly and the best known toxic furanosesquiterpenoid ketones are ngaione, epingaione, dehydrongaione, deisopropylngaione, dehydroepingaione, myomontanone, isomyomontanone, myodesmone and dehydromyodesmone. Only myoporone and its analogues were found to be less or non-toxic to the sheep. The major toxic Myoporum spp. with high content of furanosesquiterpenoid ketones are $M$. deserti, $M$. acuminatum, $M$. montanum, $M$. tenuifolium, $M$. insulare, $M$. tetrandrum, $M$. laetum, M. crassifolium, M. bontioides and M. betcheanum [46,75,78]. Myoporum toxicity is concerned mainly with the liver, kidney and lung, causing pulmonary oedema $[4,75,78]$. The highly toxic plant organs were the aerial parts including leaves, where the oil components are concentrated. The toxicity of Myoporum spp. is mainly attributed to the presence of ngaione (furanosesquiterpenoid ketones), which is responsible for the typical Myoporum toxicity signs and symptoms [78-80]. So, the Myoporum members which contain ngaione or its analogues are regarded as typical toxicants. The toxicity of Myoporum was firstly discovered and recognized when outbreaks of photosensitization and death have been developed in cattle in many Australian farms during the period (1979-1982). About 26 days after feeding on the nearest and hanging branches of $M$. insulare, the cattle have developed intoxication signs of photosensitization, depression, jaundice, petechia, anorexia and agalactia (failure of cows to produce milk). The severe intoxication was manifested by liver haemorrhage and necrosis with bile duct hyperplasia. When the experimental cows were tested for Myoporum intoxication, they have developed the same symptoms upon treatment with minced and wetted fresh leaves of $M$. insulare by oral intubation. The symptoms have been developed $72 \mathrm{~h}$ following intubation $[79,80]$. The histopathological examination of the autopsied cows showed yellow pigmentation and necrotic liver, either in periportal, midzonal or centrilobular part. Proliferation of the bile duct was also observed, and the SGT, AST and CPK enzymes were elevated [78-80]. In case of $M$. deserti and $M$. acuminatum, the symptoms of toxicity were developed $24 \mathrm{~h}$ following the feeding of the experimental cattle, while death occurred within $48 \mathrm{~h}$ after consumption [81, 82]. The degree of intoxication depending mainly on the furanosesquiterpenoid contents as well as the activity of liver microsomal mixed-function oxygenase enzymes (MMFO) during the consumption time of grazing plants, which in turn may be influenced by nutritional style of the animal $[75,76,83]$. So, intoxication may be fully or partially reduced upon inactivation of this enzyme by pretreatment with inhibitors [63, 76, 84]. Upon enhancement of liver MMFO, the toxicity may be periportal rather than centrilobular lesions [83]. From the literature survey, certain Myoporum spp. were considered extremely toxic, for example, the two varieties of $M$. deserti (Jackson \& Theodore) were classified as livestock-poisoning due to their major toxic furanosesquiterpenoid ketones; (-)-ngaione, (-)-epingaione, (-)-dehydrongaione, and (-)dehydroepingaione [29]. This toxicity was estimated by the histopathological examination of the liver and renal lesions, of the intraperitoneally injected mice with these individual sesquiterpenoid ketones [29]. Additionally, M. laetum was regarded as totally toxic plant; it causes liver injury associated with thrombosis after being consumed by cattle due to the high ngaione content [85]. The toxicity of which 
has been considered at the early stages of the last century, it was observed that when the livestock (horses, cattle, pigs and sheep) tend to feed on the leaves of hanging branches of M. laetum, severe fatal toxicity have been developed in most cases and manifested by photosensitivity, liver damage, jaundice and icterus [86-88]. Moreover, the essential oil M. tetrandrum was categorized as toxic as $M$. deserti due to the presence of dehydrongaione (78\%) and ngaione (7\%) as the main contents, its toxicity was manifested by liver damage with extensive haemorrhagic centrilobular necrosis in calves [29,83], associated with pulmonary edema in most cases [83]. With the exception of some furanosesquiterpenoids, Blackburne 1972 has reported myoporone and its derivatives as nontoxic metabolites to the sheep if orally consumed [89]. Only the I.P. injected mice with myoporone or its analogues showed ngaione-like toxicity while sheep didn't [89]. M. montanum was found to be rich in toxic furanosesquiterpenoids which are livestock poisoning. Owing to the fact that toxic furanoid members are degraded at temperature higher than $20{ }^{\circ} \mathrm{C}$ and its aqueous extract contains only $( \pm)$ Myoporone (-)-10,11-dehydromyoporone and 11-hydroxymyoporone which are benign and non-toxic to the sheep [20], the infusion-prepared tea of which has been applied safely in folk medicine by the aborigines in treatment of skin infection, otitis and dermatitis topically, and in gastritis and respiratory system orally [20].

\subsection{Mechanism of Myoporum Toxicity}

Furanosesquiterpenoid derivatives are nearly distributed in all Myoporum spp. as per the literature survey. The ketone type sesquiterpenes are believed to cause renal injury and pulmonary lesions in mice by direct tubular toxicity (Figure 2), as reported by Ogunsan 2012 for Deisopropylngaione (DIN) [90,91]. Dongju and Weiwei 2016 have proven that the presence of furan moiety in certain phytochemicals such as 8-epidiosbulbin E acetate induces hepatotoxicity $[92,93]$. Weiwei concluded that, the furanoid diosbulbin B (DIOB) compound which is the main phytochemical of Dioscorea bulbifera produced liver damage in humans and experimental animals, while the same hydrogenated tetrahydrofuran moiety of DIOB hasn't developed any toxicity. Consequently, Weiwei suggested that liver injury is attributed to the presence of furan moiety, which can be converted into reactive toxic metabolites by the cytochrome P450 3A enzymes [93]. Additionally, WU claimed that furan ring is necessary for induction of toxicity through the oxidation step by cytochrome P450 to form the reactive intermediate, which causing the toxic effects [94, 95]. Consequently, the furanosesquiterpenoids of Myoporum were supposed to induce their toxicity by the same mechanism [96-97].

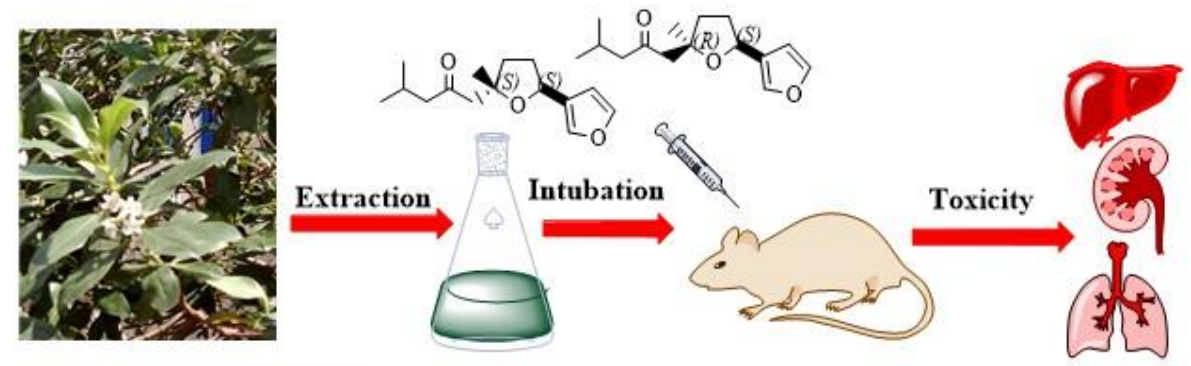

Figure 2. in vivo furanosesquiterpenoid toxicity of Myoporum spp.

\section{Conclusion}

It has become apparent that the application of plants in folk medicine is not always safe and must be cautiously used. All furanosesquiterpenoid-containing Myoporum are regarded as potentially toxic plants and can induce ngaione-like symptoms and toxicity, except myoporone and its analogues. 
Most of Myoporaceous plants, particularly those of genus Myoporum are categorized as liver, kidney and lung poisoning plants due to their furanosesquiterpenoid contents. About 32 Myoporum spp. were reported in literature, most of them haven't been resolved yet in the plant list; a standard website for listing all plant spp. Additionally, some of these species haven't been fully studied, and the investigation concerning their toxic profile is strongly recommended to save the livestock. Despite the severe toxicity of most Myoporum spp., their aqueous extract can be safely applied in folk medicine for the treatment of several diseases if prepared by infusion. Finally, the use of natural remedies doesn't convey a guarantee of safety.

\section{Acknowledgments}

The author is very grateful to Jouf University for support of this work.

\section{Conflict of interest}

The author declares that there is no conflict of interest.

\section{ORCID}

Arafa Musa: $\underline{0000-0003-0979-556 \mathrm{X}}$

\section{References}

[1] E. L. Ghisalberti (1995). The chemistry of unusual terpenoids from the genus Eremophila, in Studies in Natural Products Chemistry 15, ed: Elsevier, pp. 225-287.

[2] T. G. Tutin, V. H. Heywood, N. A. Burges and D. H. Valentine (1964). Flora Europaea: Plantaginaceae to Compositae (and Rubiaceae), 4, ed: Cambridge University Press, 190.

[3] B. Dinda (2019). Pharmacology and applications of naturally occurring iridoids: Springer, Switzerland.

[4] R. J. Chinnock (2007). Eremophila and allied genera: a monograph of the plant family Myoporaceae, $1^{\text {st }}$ ed., Rosenberg Publishing, Dural, N.S.W. 80-158.

[5] G. L. Webster (1951). The Polynesian species of Myoporum, Pac. Sci. 5(1), 55-77.

[6] S. Carlquist and D. A. Hoekman (1986). Wood anatomy of Myoporaceae: ecological and systematic considerations, Aliso: J. System. Evolut. Bot. 11, 317-334.

[7] E. L. Ghisalberti (1993). The phytochemistry of the Myoporaceae, Phytochemistry 35, 7-33.

[8] G. S. Richmond and E. L. Ghisalberti (1995). Cultural, food, medicinal uses and potential applications of Myoporum species (Myoporaceae), Econom. Bot. 49, 276-285.

[9] L. A. Mound and D. C. Morris (2007). A new thrips pest of Myoporum cultivars in California, in a new genus of leaf-galling Australian Phlaeothripidae (Thysanoptera), Zootaxa 1495, 35-45.

[10] C. Shogren (2017). Myoporum thrips life history, Doctoral dissertation, host preference, and biological control, University of California, Riverside.

[11] S. M. Walters and J. Cullen (1984). The European garden flora: a manual for the identification of plants cultivated in Europe, both out-of-doors and under glass, 6(4), Cambridge University Press, 412.

[12] T. Iwashina and G. Kokubugata (2010). Flavonoids in the leaves and flowers of Myoporum bontioides native to northernmost region in the Myoporaceae, Bull. Natl. Sci. Mus. Ser. B, 36, 117-125.

[13] C. Menut, P. Cabalion, E. Hnawia, H. Agnaniet, J. Waikedre and A. Fruchier (2005). Two new furanosesquiterpenes from Myoporum crassifolium from New Caledonia, Flav. Fragr. J. 20, 621-625.

[14] J. G. Tan, W. Dong and L.X. Qiaolin (2019). Preparation of new alkaloids as antibacterial agent for methicillin-resistant Staphylococcus aureus, China Patent CN 109553565.

[15] H. Grant, P. O'Regan, R. Park and M. Sutherland (1980). Terpenoid chemistry. XXIV.(1R)-1methoxymyodesert-3-ene, an iridoid constitutent of Myoporum deserti (Myoporaceae), Aust. J. Chem. 33, 853-878.

[16] H. Grant, C. Russell-Maynard and M. Sutherland (1985). Terpenoid chemistry. XXVII. further iridoid constituents,(1R)-and (1S)-1-acetoxymyodesert-3-ene, from Myoporum deserti (Myoporaceae), Aust. J. Chem. 38, 325-336. 
[17] L.M. Dong, L.L. Huang, H. Dai, Q.L. Xu, J.K. Ouyang, X.C. Jia, W.X. Gu and J.W. Tan (2018). AntiMRSA sesquiterpenes from the semi-mangrove plant Myoporum bontioides A. Gray, Mar. drugs, 16, 438-445.

[18] M. El-Hamouly and A. Salama (2004). Anatomical and phytochemical investigation of flowers and/or fruits of some ornamental plants, growing in Egypt, Egypt. J. Biotechnol. 16, 162-176.

[19] C. Li, B. Gong, D. G. Cox, C. Li, J. Wang and W. Ding (2014). Dichlorodiaportinol A-A new chlorinecontaining isocoumarin from an endophytic fungus Trichoderma sp. 09 from Myoporum bontioides A. Gray and its cytotoxic activity, Pharmacog. Mag. 10, S153.

[20] D. Zaleta-Pinet, A. McCluskey, S. Hall, J. Brophy, C. Ashhurst-Smith, J. Sakoff and I. V. Altena (2016). The use of the toxic plant Myoporum montanum in a traditional Australian aboriginal medicine, Aust. J. Chem. 69, 161-168.

[21] E. Flaskamp, G. Nonnenmacher and O. Isaac (1981). Zur diastereoisomerie natürlicher und synthetischer $\alpha$-bisabolole/on the diastereoisomerism of natural and synthetic $\alpha$-bisabolols, $Z$. Naturforsch. B, 36, 114-118.

[22] P. D. F. Cabalion, A. R. F. Maxime, J. K. Waikedre, C. G. F. Fourneau, C. M. Lafforgue, M. A. Vesin and E. S. Hnawia (2009). Use of an essential oil for the preparation of a cosmetic or pharmaceutical composition, France Patent FR 2922765.

[23] T. H. Johnston and J. B. Cleland (1942). Aboriginal names and uses of plants in the Ooldea region, South Australia, Transact. Royal Soc. South Austral. 66, 93-103.

[24] J.R. Weng, L.Y. Bai, W.Y. Lin, C.F. Chiu, Y.C. Chen, S.W. Chao, S.W. Chao and C.H. Feng (2017). A flavone constituent from Myoporum bontioides induces M-phase cell cycle arrest of MCF-7 breast cancer cells, Mololecules 22, 472-484.

[25] Q.G. Wang, C.L. Ma and J.J. Zhai (2000). Furanoeudesmane-B, a new eudesmane sesquiterpenoid from Myoporum bontioides, Acta Crystallogr. Sect. C: Crys. Struct. Comm. 56, iuc0000293-e569.

[26] D. Yecheng, Y. Zhen, Y. Yanzhen and B. Xiulian (2008). Inhibitory activity against plant pathogenic fungi of extracts from Myoporum bontioides A. Gray and indentification of active ingredients, Pest Managem. Sci. 64, 203-207.

[27] A. A. Zaki, M. I. Shaaban, N. E. Hashish, M. A. Amer and M.F. Lahloub (2013). Assessment of antiquorum sensing activity for some ornamental and medicinal plants native to Egypt, Scient. Pharmaceut. 81, 251-258.

[28] L. Hu, Y. Guo and W. Gu (2013). Flavonoids from the leaves of Myoporum bontioides, J. Trop. Subtrop. Bot.21, 266-272.

[29] W. Hamilton, R. Park, G. Perry and M. Sutherland (1973). Terpenoid chemistry. XXI.(-)-epingaione,()-dehydrongaione,(-)-dehydroepingaione and (-)-deisopropylngaione, toxic furanoid sesquiterpenoid ketones from Myoporum deserti," Aust. J. Chem. 26, 375-387.

[30] H. Brockman (2016). Renewable chemicals and bioproducts: a potential for agricultural diversification and economic development, Department of Agriculture and Food, Western Australia, Perth.

[31] M. Tawfik, W. M. Haggag, E. G. Mirvat, M. Kabish and S. El Habbasha (2015). Determination of nutritional value and lignocellulosic biomass of six halophytic plants grown under saline irrigation in South Sinai, Int. J. ChemTech Res. 8, 37-42.

[32] L. Xianzhen, L. Chunyuan, G. Wenxiang and W. Zhixiong (2010). Isolation, identification and bioassay of myoporone from the volatile oil of Myoporum Bontiodes, Guang. Chem. Ind. (37)9, 9-10.

[33] H.L. Lan, L.J. Wen, N.C. Lin, G.W. Xiang and L.C. Yuan (2011). Isolation, crystal structure and inhibitory activity against Magnaporthe Grisea of (2R, 3R)-3, 5, 7-trihydroxyflavanone 3-acetate from Myoporum Bontioides A. Gray," Chin. J. Struct. Chem. 30, 1298-1304.

[34] M. T. I. Salama (2017). Antimicrobial activity of essential oil of Myoporum acuminatum R. Br fruits, cultivated in Libya, J. Essent. Oil-Bear. Plants. 20, 233-239.

[35] C. C. Duke, R. K. Duke and V. H. Tran (2016). Medicinal use of serrulatane diterpenes, Australian Patent: AU 2016903585

[36] G. Liu (2014). A herbal composition for treating sciatica, China Patent: CN 104116834.

[37] Y. Wu (2014). A medicine composition for treatment of intractable headache, China Patent:CN 104116932.

[38] D. Yang (2013). Chinese medical composition for treating upper respiratory tract infection and its formulation, China Patent:CN 103446348.

[39] N. Ibrahim, S. Mohamed, M. Faraid and E. Hassan (2006). Chemical composition, antiviral and antimicrobial activities of the essential oils of Aster novi-belgii, Solidago canadensis and Myoporum laetum growing in Egypt, Bull. Fac. Pharm. Cairo Univ. 44, 103-110.

[40] S. M. Mohamed and E. A. E.F. Omer (2009). Seasonal variation in the volatile oil of Myoporum laetum leaves, Aust. J. Chem. 25, 1779-1786. 
[41] E.H. M. and S. A. (2004). Anatomical and phytochemical investigation of flowers and/or fruits of some ornamental plants, growing in Egypt," Egypt J. Biotech. 16, 162-176.

[42] T. Kubota and T. Matsuura (1958). On the constitution of myoporone (natural furan derivatives. II.), Bull. Chem. Soci. Japan, 31, 491-494.

[43] T. T. Minh, T. T. Ngoan, N. T. M. Thuong, H. K. Toan, N. X. Truong, T. T. Huong, G. T. P. Ly D. T. T. Ai and N. D. Khanh (2020). Chemical compositions and bioefficacy against Spodoptera litura of essential oil and ethyl acetate fraction from Myoporum bontioides leaves, Vietnam J. Chem. 58, 57-62.

[44] M. Kanemoto, K. Matsunami, H. Otsuka, T. Shinzato, C. Ishigaki and Y. Takeda (2008). Chlorinecontaining iridoid and iridoid glucoside and other glucosides from leaves of Myoporum bontioides, Phytochemistry 69, 2517-2522.

[45] F. Tomas, F. Ferreres, F. Barberan and J. Nieto (1985). Flavonoid diglycosides from Myoporum tenuifolium, J. Nat. Prod. 48, 506-507.

[46] P. L. Métra and M. D. Sutherland (1983). Further skeletal variety in the toxic furanosesquiterpene ketones in the Myoporum genus, Tetrahedron Lett. 24, 1749-1752.

[47] R. Hegenauer and M. Hegenaue (1964). Chemotaxonomie der Pflanzen Bd-3, ed: IX, Basel-Stuttgart.

[48] R. Kadirvelraj, P. N. Handley, W. T. Robinson, R. M. Carman and M. D. Sutherland (1999). The stereochemical structures of the furanosesquiterpenoidal $\beta$-hydroxy ketones from the Myoporaceae, Aust. J. Chem. 52, 727-732.

[49] K. O'Brien, A. Penfold M. Sutherland and R. Werner (1954). The sesquiterpene alcohol of Myoporum crassifolium Forst, Aust. J. Chem. 7, 298-300.

[50] M. T. Estan (1985). Diglycosylamines in Myoporum tenuifolium (E. Foster)," Rev. Agroquim. Tecnol. Aliment. 25, 369-72.

[51] F. Barberan, M. Gil, F. Tomas and F. Ferreres (1985). Polyphenolic compounds from the leaves of Myoporum tenuifolium, Plants Med. Phytother. 19, 48-56.

[52] M. Estan (1985). Glucosids of flavonoids in Myoporum tenuifolium (E. Foster), Rev. Agroquim. Tecnol. Aliment. 25, 273-278. 1985.

[53] H. Ye, H. Dai, L. Wu, Y. Guo and W. Gu (2014). Chemical constituents from leaves of Myoporum bontioides and their bacteriostatic activities, J. Trop. Subtrop. Bot. 22, 307-313.

[54] H.L.J. Wen, N. C. W. Xiang and L.C. Yuan (2011). Isolation, crystal structure and inhibitory activity against magnaporthe grisea of (2R, 3R)-3, 5, 7-trihydroxyflavanone 3-acetate from Myoporum Bontioides A. Gray, Chin. J. Struct. Chem. 30,1298-1304.

[55] M. F. Lahloub, S. H. El Sharkawy, M. M. Ahmad and A. A. Galala (2003). Chemical study of Myoporum laetum Forst. f.," Mansoura J. Pharm. Sci. 19, 73-89.

[56] N. Aminimoghadamfarouj and A. Nematollahi (2017). Structure elucidation and botanical characterization of diterpenes from a specific type of bee glue, Molecules 22, 1185-1198.

[57] J. H. Langenheim (2004). Plant resins: chemistry, evolution, ecology and ethnobotany, Ann. Bot. 93, 784-785.

[58] M. El-Hamouly, H. Ammar and A. Awaad (2001). Essential oil composition and antimicrobial activity of Myoporum acuminatum (G.) Foster, cultivated in Egypt, Az. J. Pharm. Sci. 28, 360-366.

[59] V. Roussis and D. Hubert (1992). Total synthesis of (+)-myomontanone and (+)-10,11didehydromyomontanone, Liebig Annal. Chem. 5, 539- 541.

[60] R. A. Massy-Westropp and G. D. Reynolds (1966). Eremophilane sesquiterpenes from Eremophila mitchelli, Aust. J. Chem.19(2), 303-307.

[61] H. Tingyu, G. Wenxiang, M. Liping, F. Liping and H. Yanbiao (2005). Studies on the chemical constituents of volatile oil from Myoporum bontiodes, J. South China Agric. Univ. 26, 114-116.

[62] I. Blackburne and M. Sutherland (1972). Terpenoid chemistry. XIX. dehydro-and dehydroisomyodesmone, toxic furanoid sesquiterpene ketones from Myoporum deserti, Aust. J. Chem. 25, 17791786.

[63] I. Blackburne, R. Park and M. Sutherland (1971). Terpenoid chemistry. XVIII. Myodesmone and isomyodesmone, toxic furanoid ketones from Myoporum deserti and M. acuminatum, Aust. J. Chem. 24, 995-1007.

[64] M. Sutherland and J. Rodwell (1989). Terpenoid chemistry. XXVIII. Furanosesquiterpene $\beta$-ketols from Myoporum betcheanum, M. deserti, M. montanum and other Myoporaceae, Aust. J. Chem. 42, 1995-2019.

[65] R. K. Murray, D. K. Granner, P. A. Mayes and V. W. Rodwell (2014). Harper's illustrated biochemistry, $30^{\text {th }}$ ed, Mcgraw-Hill Education, Willey.

[66] L. Sibulesky (2013). Normal liver anatomy, Clinic. Liv. Dis. 2(S1), S1-S3.

[67] W. J. Welch (1992). Mammalian stress response: cell physiology, structure/function of stress proteins and implications for medicine and disease, Physiol. Rev. 72, 1063-1081. 
[68] U. J. Tietge, K. H. Boker, M. P. Manns and M. J. Bahr (2004). Elevated circulating adiponectin levels in liver cirrhosis are associated with reduced liver function and altered hepatic hemodynamics, Amer. J. Physiol. Endocrin. Metabol. 287, E82-E89.

[69] A. M. Diehl (2002). IV. Nonalcoholic fatty liver disease abnormalities in macrophage function and cytokines, Amer. J. Physiol.Gastrointest. Liv. Physiol. 282, G1-G5.

[70] T. M. Saleem, C. M. Chetty, S. Ramkanth, V. Rajan, K. M. Kumar and K. Gauthaman (2010). Hepatoprotective herbs-a review, Int. J. Res. Pharm. Sci. 1, 1-5.

[71] N. Anderson, R. Gama and S. Kapadia (2001). Herbal remedy poisoning presenting with acute abdomen and raised urine porphyrins, Ann. Clinic. Biochem. 38, 408-410.

[72] E. Björnsson (2010). Drug-induced liver injury in clinical practice, Aliment. Pharmacol. Therap. 32, 313.

[73] A. Seawright and J. Hrdlicka (1972). The effect of prior dosing with phenobarbitone and $\beta$ diethylaminoethyl diphenylpropyl acetate (SKF525A) on the toxicity and liver lesion caused by ngaione in the mouse, Br. J. Exp. Path. 53, 242-252.

[74] R. F. Keeler, K. R. Van Kampen and L. F. James (2013). Effects of poisonous plants on livestock, Elsevier.

[75] A. Seawright, J. Hrdlicka, J. Lee and E. Ogunsan (1982). Toxic substances in the food of animals: Some recent findings of Australian poisonous plant investigations, J. App. Toxicol. 2, 75-82.

[76] A. Seawright, J. Lee, J. Allen and J. Hrdlicka (1978). Toxicity of Myoporum spp. and their furanosesquiterpenoid essential oils, in Effects of Poisonous Plants on Livestock, ed: Elsevier, 241-250.

[77] P. D. Constable, K. W. Hinchcliff, S. H. Done and W. Grünberg (2016). Veterinary medicine-e-book: a textbook of the diseases of cattle, horses, sheep, pigs and goats: Elsevier Health Sciences.

[78] M. G. Maxie (2016). Liver and biliary system, Jubb, Kennedy \& Palmer's Pathology of Domestic Animals, ed: Elsevier.

[79] I. Jerrett and R. Chinnock (1983). Outbreaks of photosensitisation and deaths in cattle due to Myoporum aff. insulare R. Br. toxicity, Aust. Vet. J. 60(6), 183-186.

[80] A. Alkhaldi, A. Musa and E. M. Mostafa P D. Koning (2020). Docking studies and antiprotozoal activity of secondary metabolites isolated from Scrophularia syriaca Benth. growing in Saudi Arabia, Rec. Nat. Prod. 14(1), 23-30.

[81] R. Riek and S. Wright (1946). The toxic properties of the volatile Oil of Myoporum acuminatum R. Br, Aust. Vet. J. 22, 149-153.

[82] A. Musa, N. S. Al-muaikel and M. S. Abdel-Bakky (2016). Phytochemical and pharmacological evaluations of ethanolic extract of Bassia eriophora, Der Pharm. Chemi. 8(12), 169-178.

[83] J. Allen, A. Seawright and J. Hrdlicka (1978). The toxicity of Myoporum tetrandrum (Boobialla) and myoporaceous furanoid essential oils for ruminants, Aust. Vet.J., 54, 287-292.

[84] A. Seawright, J. Lee, J. Allen and J. Hrdlicka (1978). Toxicity of Myoporum spp. and their furanosesquiterpenoid essential oils, in Joint United States-Australian Symposium on Poisonous Plants, Logan, Utah (USA).

[85] S. Al-Qura'n (2005). Ethnobotanical survey of folk toxic plants in southern part of Jordan, Toxicon 46, 119-129.

[86] F. Denz and W. Hanger (1961). The liver toxin in Myoporum laetum, J. Pathol. Bacteriol. 81, 91-99.

[87] J. Raposo, M. Méndez, F. Riet-Correa and G. De Andrade (1998). Experimental intoxication by Myoporum laetum in sheep, Vet. Hum. Toxicol., 40, 132-135.

[88] J. Raposo, M. d. C. Mendez and F. Riet-Correa (1998). Experimental intoxication by Myoporum laetum in cattle, Vet. Hum. Toxicol. 40, 275-277.

[89] I. Blackburne, R. Park and M. Sutherland (1972). Terpenoid chemistry. XX. Myoporone and dehydromyoporone, toxic furanoid ketones from Myoporum and Eremophila species, Aust. J. Chem. 25, 1787-1796.

[90] N. Sadgrove, G. L. Jones, B. Greatrex and K. Watson (2014). Ethnopharmacology and chemotaxonomy of essential oil yielding Australian plants, doctoral thesis, University of New England Australia.

[91] A. A. S. E.A. Ogunsan, D.O. Ehizibolo, H.M. Tukur, M.S. Ahmed, Y.G. Dashe and M.Y. Sugun, (2012). The acute toxicity of deisopropylngaione in mice, Kid. Res. J. 2, 12-17.

[92] D. Lin, W. Li, Y. Peng, C. Jiang, Y. Xu, H. Gao and J. Zheng (2016). Role of metabolic activation in 8epidiosbulbin E acetate-induced liver injury: mechanism of action of the hepatotoxic furanoid, Chem. Res. Toxicol. 29, 359-366.

[93] W. Li, D. Lin, H. Gao, Y. Xu, D. Meng, C. V. Smith, Y. Peng and J. Zhenget (2016). Metabolic activation of furan moiety makes Diosbulbin B hepatotoxic, Arch. Toxicol. 90, 863-872. 
[94] W. Zi-Tian, L.Z. Qing, S. Wei, W. L. Li, Y. Jiang, L. Ping and L.H. Jun (2020). The crucial role of metabolic regulation in differential hepatotoxicity induced by furanoids in Dioscorea bulbifera, Chin. J. Nat. Med. 18, 57-69.

[95] X. Cachet, J. Langrand, L. Riffault-Valois, C. Bouzidi, C. Colas, A. Dugay, S. Michel and D. BoucaudMaitre (2018). Clerodane furanoditerpenoids as the probable cause of toxic hepatitis induced by Tinospora crispa, Scientific Rep. 8, 1-11.

[96] M. M. Ghoneim, A. Musa, A. A. El-Hela and K. M. Elokely (2018). Evaluation and understanding the molecular basis of the antimethicillin-resistant Staphylococcus aureus activity of secondary metabolites isolated from Lamium amplexicaule, Pharmacog. Mag. 14 (55), S3-S7.

[97] A. Musa (2019). Chemical constituents, antimicrobial and antiinflammatory evaluations of various extracts of Suaeda vera Forssk. growing in Saudi Arabia, Int. J. Pharm. Res. 11(4), 962-967.

\section{A C G \\ publications \\ (C) 2020 ACG Publications}

